\title{
Analyses of Semiconductor Materials by Spectrometric Full-color Cathodoluminescence Microscopy
}

\author{
H. Saijo, Y. Suzuki* and M. Shiojiri** \\ Faculty of Biology Oriented Science and Technology, Kinki University, 930 Nishimitani, Kinokawa, \\ Wakayama, 649-6493, Japan, *Graduate School, Biology Oriented Science and Technology, Kinki \\ University, 930 Nishimitani, Kinokawa, Wakayama, 649-6493, Japan, **Prof. Emeritus, Kyoto Institute \\ of Technology, 1-297 Wakiyama, Ohyamazaki, Kyoto, 618-0091, Japan
}

The luminescence released by a photon incidence, so-called photoluminescence (PL), provides information of electronic states of the specimen such as the band gap of semiconductors or the highest occupied molecular orbital (HOMO)-lowest unoccupied MO (LUMO) of organic materials. However, the band-gap of recent semiconductor materials for optoelectronic devices is as wide as several $\mathrm{eV}$, and the excitation of such materials becomes impossible by regular light source including discharge lamps for PL study use. Focusing and scanning of probing photons on the specimen contains another difficulty if microscopic observation is tried by PL microscopy. The luminescence upon electron incidence, cathodoluminescence (CL) can overcome all above difficulties. Our new spectrometric full-color CL microscope [1] collects CL spectra of each observing point, and draws full-color CL and corresponding secondary electron micrographs of 512 x 512 pixels in $8 \mathrm{~s}$, which enabled us to observe irradiationsensitive materials such as organic or biological materials. Since CL is excited by the incident narrowfocused electron beam in SEM, most electronic states are excited and luminescence light down to $200 \mathrm{~nm}$ wavelength can be released. Spatial resolution of CL micrographs may become worse than that of SEM due to scattering of incident electrons in the specimen. Delayed CL emission is another cause of poor resolution. Both result in practical CL resolution around $100 \mathrm{~nm}$. By choosing lower incident energy and slow image scan speed, the spatial resolution of CL observation is significantly improved, and in our system $25 \mathrm{~nm}$ resolution was verified with $6 \mathrm{kV}$ beam and $40 \mathrm{~s} /$ frame. In the present study, we summarize previous studies and show new observations on growth and qualification of GaN/InGaN layers for laser diode fabricated by metalorganic vapor-phase epitaxy (MOVPE) method [2], deep-UV emission from grain boundaries of $\mathrm{ZnO}$ varistor [3], and cyanine dye $\mathrm{J}$-aggregates. The block diagram of the system is shown in Fig. 1. In the SEM image of Fig. 2, we noticed two white lines (pointed by green arrows and noted as " $D$ " $\left(D_{1}, D_{2}\right)$ in the corresponding CL image) run. They are assumed to be defects being created on the substrate surface upon epitaxy and kept growth across hetero interfaces of InGaN/GaN layers. CL spectra along the white lines show sharp emission at about $560-570 \mathrm{~nm}$ that is absent in the spectra of other parts marked by "N" $\left(\mathrm{N}_{1}-\mathrm{N}_{3}\right)$. Hence, the $560-570 \mathrm{~nm}$ peak is characteristic to the defect structure. A survey of CL spectra throughout the specimen revealed another points of the characteristic $560-570 \mathrm{~nm}$ peak (marked by "H"). In the SEM, no special contrast is seen along " $\mathrm{H}$ ", which suggests the defect lying deep from the surface. Luminescence light can escape deep from the surface whereas secondary electrons hardly do. Fig. 4 is a study of grain boundaries of $\mathrm{ZnO}$ varistor [3]. CL observation across the boundary revealed that, from the boundary, $500 \mathrm{~nm}$ peak appears first, then $510 \mathrm{~nm}$ peak rises slightly inside 


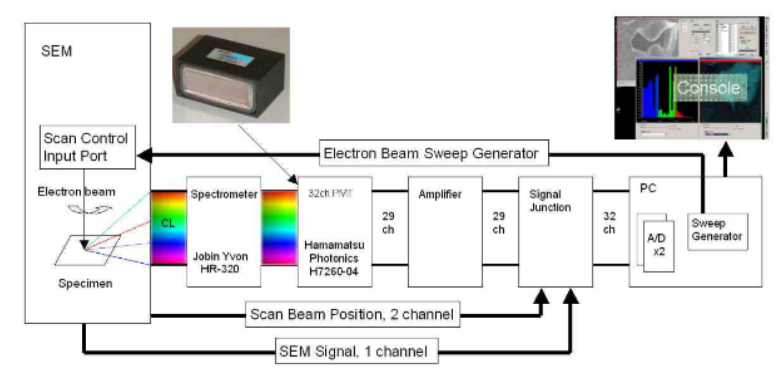

Fig. 1. System block diagram of spectrometric full-color cathodoluminescnece microscope.

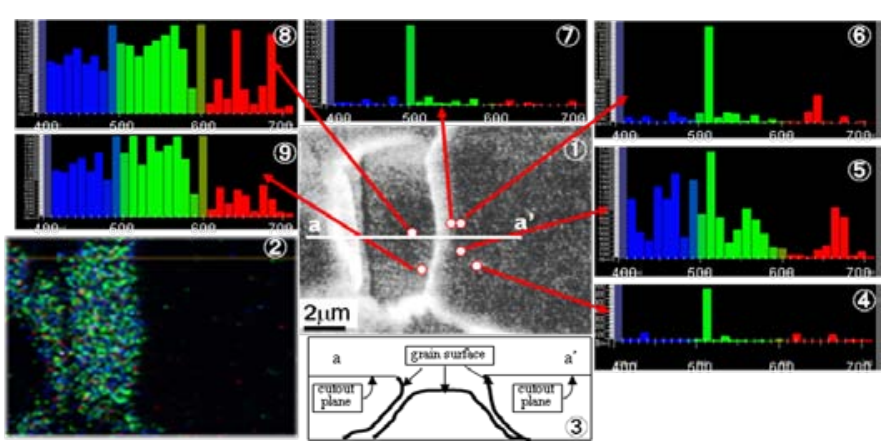

Fig. 4. Spectral variation near the grain boundaries of $\mathrm{ZnO}$ varistor. 1 and 2: SEM and full color CL micrograph. 3: Schematic diagram of the cross section, a-a'.4-9: CL spectra of points marked in 1.

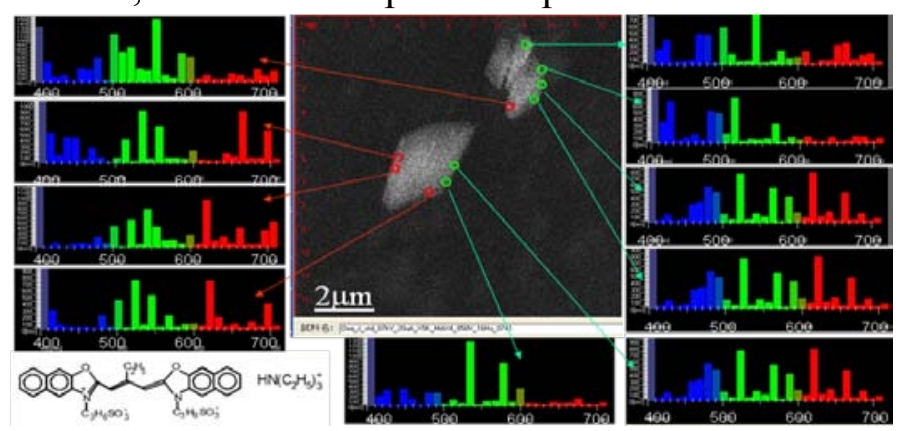

Fig. 5 Spectral variation of CL from Oxacarbocyanine J-aggregates grown in water.

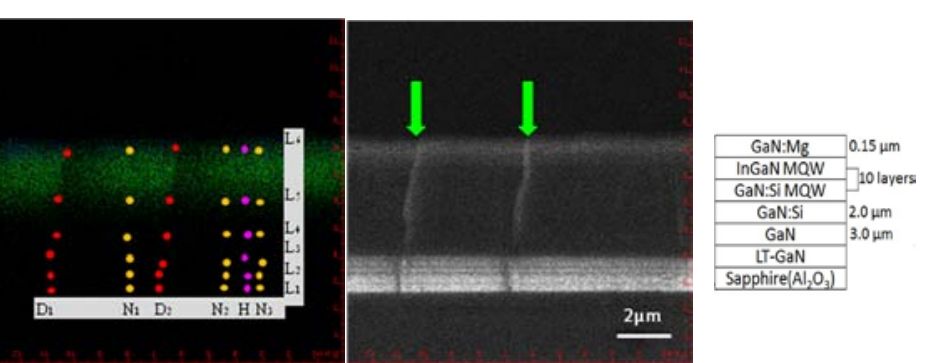

Fig. 2. CL and SE images of InGaN/GaN wafer. Color dots are the points of CL spectra in Fig. 3

N1

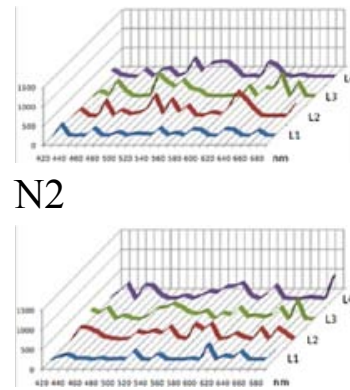

N3

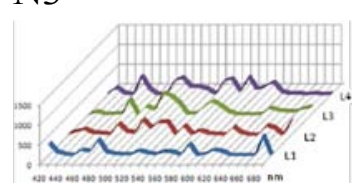

D1

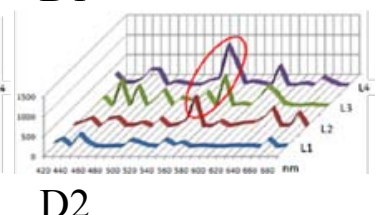

D2

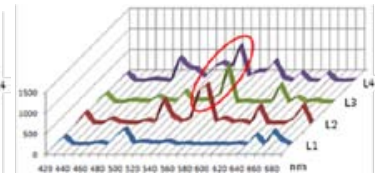

$\mathrm{H}$

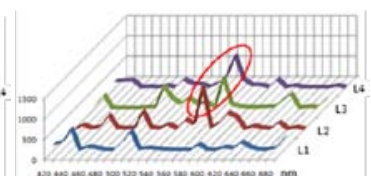

Fig. 3. Spectral variation of layers $\mathrm{L}_{1}, \mathrm{~L}_{2}, \mathrm{~L}_{3}$ and $\mathrm{L}_{4}$ at columns $\mathrm{N}_{1}, \mathrm{H}, \mathrm{N}_{2}, \mathrm{D}_{1}, \mathrm{~N}_{3}$ and $\mathrm{D}_{2}$ the grain. Then, $490 \mathrm{~nm}$ peak twins with 510 $\mathrm{nm}$ peak. The spectral variation suggests the change of elemental composition across the boundary. This material also emits deep-UV luminescence at about $210 \mathrm{~nm}$ [5].

Cyanine dye molecules gather taking special arrangement each other and form so-called Jaggregates, and the aggregate emits sharp and strong luminescence. Fig. 5 shows the spectral variation of CL depending on the position and angle of dye molecules inside aggregates.

Our new spectrometric full-color CL microscope showed successfully the ability of detection such as hidden defects and structural variation near boundaries, and heterogeneous luminescence emission.

References

1. H. Saijo and M. Shiojiri, "Proc. 16th IMC", Vol. 2, (2006) 883., Mat. Trans., 51 (2010), 231-236.

2. Kindly provided by Dr. J.T. Hsu, Ind. Technol. Res. Inst., Hsinchu, Taiwan.

3. Kindly prepared by Daneu N and Recnik A, Jozef Stefan Inst., Ljubljana, Slovenia

4. H. Saijo, N. Daneu, A. Recnik, and M. Shiojiri, in "Proc. EMC2008. Aachen", (2008). 1192.

5. H. Saijo, Y. Suzuki and M. Shiojiri; J. Phys., Conference Series, to be published. 